\title{
Diagnóstico situacional de uma Unidade Básica de Saúde: um relato de experiência
}

\author{
Situational diagnosis of a Basic Health Unit: an experience report \\ Diagnóstico situacional de una Unidad Básica de Salud: relato de experiencia
}

Ana Klara Rodrigues Alves

ORCID: https://orcid.org/0000-0002-1216-9386 Universidade Estadual do Piauí, Brasil E-mail: klaraphb@outlook.com

Barbara Beatriz Lira da Silva

ORCID: https://orcid.org/0000-0001-9166-6147 Universidade Estadual do Piauí, Brasil E-mail: brbeatriz16@gmail.com

Larissa dos Santos Silva

ORCID: https://orcid.org/0000-0001-8485-4094 Universidade Estadual do Piauí, Brasil E-mail: lari.enf10@gmail.com

Senira de Oliveira Rodrigues Lavor ORCID: https://orcid.org/0000-0002-5225-1034 Universidade Estadual do Piauí, Brasil E-mail:seniraoliveira@phb.uespi.br

Jardel Silva Santos

ORCID: https://orcid.org/0000-0002-9615-6864 Universidade Estadual do Piauí, Brasil E-mail: jardelsilva40.eng@gmail.com

Fábio Dias Nogueira

ORCID: https://orcid.org/0000-0001-7303-1180 Faculdade de Ciências Humanas, Exatas e da Saúde do Piauí, Brasil E-mail: fdnvaq@gmail.com

Francisco das Chagas da Silva Serejo Júnior ORCID: https://orcid.org/0000-0002-5167-2830 Faculdade de Ciências Humanas, Exatas e da Saúde do Piauí, Brasil E-mail: fserejojunior@hotmail.com

Renan Silva Galeno

ORCID: https://orcid.org/0000-0001-9607-9590 Faculdade de Ciências Humanas, Exatas e da Saúde do Piauí, Brasil E-mail: renangaleno@gmail.com

Helder Marques Lima Júnior

ORCID: https://orcid.org/0000-0003-0129-1279 Faculdade de Ciências Humanas, Exatas e da Saúde do Piauí, Brasil E-mail: heldermljr@hotmail.com

Edmar José Fortes Júnior

ORCID: https://orcid.org/0000-0002-0114-9937 Faculdade de Ciências Humanas, Exatas e da Saúde do Piauí, Brasil E-mail: edmarfortes@hotmail.com

Alvaro Martins Pinho

ORCID: https://orcid.org/0000-0002-2560-4674 Faculdade de Ciências Humanas, Exatas e da Saúde do Piauí, Brasil

E-mail: alvaromartinspinho@ hotmail.com

Lívia Filomena Castelo Branco Machado ORCID: https://orcid.org/0000-0001-5073-8140 Centro Universitário UNINOVAFAPI, Brasi E-mail: liviafilomenacbm@hotmail.com

Maria de Lourdes Pereira Ibiapina ORCID: https://orcid.org/0000-0001-7444-1245 Centro Universitário UNINOVAFAPI, Brasi E-mail: maluibiapina14@gmail.com

Fábio Landel Alysson Araújo Franklin ORCID: https://orcid.org/0000-0003-0490-3701 Centro Universitário UNINOVAFAPI, Brasil E-mail: flandel5@hotmail.com

Lia Moreira de Vasconcelos Almeida ORCID: https://orcid.org/0000-0002-6595-9613 Centro Universitário UNINOVAFAPI, Brasil E-mail: liamvalmeida@gmail.com 


\begin{abstract}
Resumo
O presente estudo realizou o diagnóstico situacional de uma Unidade Básica de Saúde (UBS), esta última é definida como conjunto de ações de saúde, no âmbito individual e coletivo, que abrange a promoção e a proteção da saúde, a prevenção de agravos, o diagnóstico, o tratamento, a reabilitação, a redução de danos e a manutenção da saúde com o objetivo de realizar o Diagnóstico Situacional de uma Unidade Básica de Saúde para avaliar o tipo de perfil populacional. Trata-se de um relato de experiência de abordagem crítico-reflexivo de cunho descritivo-compreensivo sobre o Diagnóstico Situacional da Unidade Básica de Saúde do bairro Ilha Grande de Santa Isabel mód.1, no município de Parnaíba-Piauí, de maio/2021 a agosto/2021. É um estudo exploratório-descritivo e diagnósticoavaliativo, onde o instrumento de pesquisa utilizado foi o Prontuário Eletrônico do Cidadão (PEC) do e-SUS. A experiência na realização do diagnóstico situacional, apresentou benefícios para formação acadêmica, pois, ajuda a desenvolver um olhar crítico e reflexivo da realidade vivenciada em uma UBS a partir de vivências teóricas na universidade. O diagnóstico situacional é um meio importante de avaliação das Unidades Básica de Saúde sendo de total necessidade na formação acadêmica, pois proporciona o entendimento dos problemas e as necessidades sociais como: necessidade de saúde, educação, saneamento, segurança, transporte, habitação, além de compreender a organização dos serviços de saúde. Indispensável para levantamento de problemas e o planejamento estratégico situacional que permite desenvolver ações de saúde mais focais efetivas em relação aos problemas encontrados.
\end{abstract}

Palavras-chave: Atenção básica à saúde; Diagnóstico; Prevenção.

\begin{abstract}
This study developed a situational diagnosis of a Basic Health Unit (UBS), which is defined as a set of health actions, in the individual and collective scope, which covers health promotion and protection, disease prevention, diagnosis, treatment, rehabilitation, harm reduction and health maintenance, with the objective of making the Situational Diagnosis of a Basic Health Unit to evaluate the type of population profile. This is an experience report of criticalreflective approach, through a descriptive-comprehensive method, about the Situational Diagnosis of the Basic Health Unit located in Ilha Grande de Santa Isabel, mod.1, in Parnaíba-Piauí, from May, 2021 to August, 2021. It is an exploratory-descriptive and diagnostic-evaluative study, which has used as research instrument the Electronic Citizen's Medical Record (PEC) from the e-SUS. The experience through the situational diagnosis development, presented benefits for academic formation, as it helps to develop a critical and reflective look at the reality experienced in an UBS from theoretical experiences at the university. Situational diagnosis is an important means of evaluating Basic Health Units, is absolutely necessary for the formation of college students, as it provides understanding of social problems and needs such as: health needs, education, sanitation, security, transport, housing, as well as allowing to know how the health services are organized, being Indispensable to know the problems and strategic situational planning that allows the development of more focused effective health actions related to the problems found.
\end{abstract}

Keywords: Basic health care; Diagnosis; Prevention.

\title{
Resumen
}

El presente estudio realizó el diagnóstico situacional de una Unidad Básica de Salud (UBS), siendo esta última definida como un conjunto de acciones de salud, en el ámbito individual y colectivo, que abarcan la promoción y protección de la salud, la prevención de lesiones, el diagnóstico, tratamiento, rehabilitación, reducción de daños y mantenimiento de la salud con el fin de realizar el Diagnóstico Situacional de una Unidad Básica de Salud para evaluar el tipo de perfil poblacional. Se trata de un relato de experiencia de abordaje crítico-reflexivo descriptivointegral sobre el Diagnóstico Situacional de la Unidad Básica de Salud del barrio Ilha Grande de Santa Isabel mod.1, en el municipio de Parnaíba-Piauí, de mayo / 2021 a agosto 2021. Se trata de un estudio exploratorio-descriptivo y diagnóstico-evaluativo, donde el instrumento de investigación utilizado fue el Registro Ciudadano Electrónico (PEC) del e-SUS. La experiencia en la realización del diagnóstico situacional tuvo beneficios para la formación académica, ya que ayuda a desarrollar una mirada crítica y reflexiva sobre la realidad vivida en una UBS a partir de experiencias teóricas en la universidad. El diagnóstico situacional es un medio importante de evaluación de las Unidades Básicas de Salud y es absolutamente necesario en la formación académica, ya que permite comprender problemas y necesidades sociales como: necesidad de salud, educación, saneamiento, seguridad, transporte, vivienda, además de comprender la organización de los servicios de salud. Indispensable para la evaluación de problemas y la planificación estratégica situacional que permita el desarrollo de acciones de salud efectivas más focalizadas en relación a los problemas encontrados.

Palabras clave: Atención sanitaria básica; Diagnóstico; Prevención.

\section{Introdução}

O Sistema Único de Saúde (SUS) é um dos maiores e mais complexos sistemas de saúde pública do mundo, abrangendo desde o simples atendimento para avaliação da pressão arterial, por meio da Atenção Primária, até o transplante de órgãos, garantindo acesso integral, universal e gratuito para toda a população do país. Com a sua criação, o SUS proporcionou 
o acesso universal ao sistema público de saúde, sem discriminação. A atenção integral à saúde, e não somente aos cuidados assistenciais, passou a ser um direito de todos os brasileiros, desde a gestação e por toda a vida, com foco na saúde com qualidade de vida, visando à prevenção e a promoção da saúde (Sistema, 2020).

O SUS é fruto de luta popular e social por um sistema de saúde que atendesse de forma igualitária, universal e equitativa a população brasileira. Dessa forma, o maior objetivo era o de proporcionar um nível de saúde para todos, independente das diferentes origens sociais e econômicas, propondo uma nova abordagem na organização e racionalização dos recursos disponíveis através dos cuidados primários à saúde (Paim, 2018).

A definição de Atenção Básica $(\mathrm{AB})$ é oferecer serviços de primeiro contato do paciente com o sistema de saúde, direcionados a cobrir as afecções e condições mais comuns e resolver a maioria dos problemas de saúde de uma população. Caracteriza-se por um conjunto de ações de saúde, no âmbito individual e coletivo, que abrangem a promoção e a proteção da saúde, a prevenção de agravos, o diagnóstico, o tratamento, a reabilitação e a manutenção da saúde. Orienta-se pelos princípios da universalidade, da acessibilidade e da coordenação do cuidado, do vínculo e continuidade, da integralidade, da responsabilização, da humanização, da equidade e da participação social (Ramos et al., 2018; Brasil, 2017).

No Brasil, a Atenção Primária constitui-se por Centros de Saúde também chamados de Unidades Básicas de Saúde (UBS) ou Postos de Saúde; seu corpo profissional conta com equipes especializadas, Equipe de Saúde da Família (ESF), para atender seus pacientes de forma a ampliar resolutividades e diminuir impactos na situação de saúde da população, reduzindo chances de encaminhá-los para a Atenção Secundária equivocadamente. Para que todo o serviço aconteça de forma eficiente, é necessário que exista uma gestão consciente de sua equipe e das peculiaridades e necessidades da região (Brasil, 2017; Tasca, Massuda, Carvalho, Buchweitz \& Harzheim, 2020).

A Ilha Grande de Santa Isabel, é um dos bairros que se localiza na cidade de Parnaíba-PI. Lá se tem o acesso da Praia Pedra do Sal e da cidade de Ilha Grande do Piauí (Morros da Mariana). Esse é um dos bairros que apresenta o maior número de cultura da cidade de Parnaíba, Bumba-meu-boi (Novo Fazendinha), Grupos Culturais de Dança (Raízes do Nordeste) e outros, além de artesanato e uma hospitalidade que não tem igual. A ligação do Centro da Cidade de Parnaíba ao Bairro de Ilha Grande de Santa Isabel (Fazendinha) se faz com um dos monumentos históricos da cidade, que é a Ponte de Simplício Dias da Silva, onde passa o Rio Igaraçu, braço direito do Rio Parnaíba. A Ilha Grande de Santa Isabel divide-se em quatro comunidades, Fazendinha (Centro da Ilha Grande de Santa Isabel), Vazantinha, Alto do Moreno e Bairro Vermelho.

O presente estudo tem como objetivo realizar o Diagnóstico Situacional de uma Unidade Básica de Saúde para avaliar o tipo de perfil populacional.

\section{Metodologia}

Trata-se de um relato de experiência de abordagem crítico-reflexivo de cunho descritivo-compreensivo sobre o Diagnóstico Situacional da Unidade Básica de Saúde do bairro Ilha Grande de Santa Isabel mód.1, no município de ParnaíbaPiauí, de maio/2021 a agosto/2021. É um estudo exploratório-descritivo e diagnóstico-avaliativo, onde o instrumento de pesquisa utilizado foi o Prontuário Eletrônico do Cidadão (PEC) do e-SUS AB, de acordo com Gil (2007), um estudo descritivo tem a finalidade de apresentar as características de uma determinada população ou de um fenômeno, com o objetivo de conhecer a área de abrangência do Centro de Saúde; identificar o perfil da comunidade que é assistida; descrever a estrutura física, e recursos humanos da UBS. A amostra da pesquisa é do tipo intencional, selecionada, entre a população definida e circunscrita das famílias cadastradas como usuárias na UBS.

Também foram utilizados artigos para agregar essa redação, encontrados na base de dados Pubmed, por meio da combinação dos descritores "Diagnóstico situacional" e "Unidade Básica de Saúde". 


\section{Resultados e Discussão}

A fim de melhor relatar essa experiência, organizamos este momento do estudo em dois tópicos: "Diagnóstico situacional da UBS", onde serão descritos os profíssionais e estrutura física; e "Descrição da população atendida na UBS", onde serão expressas as limitações, dificuldades, e a percepção em relação à contribuição da pesquisa no crescimento acadêmico para a formação do sujeito coletivo na inserção da investigação científica entre os impactos da experiência, pode-se citar a produção de benefícios para formação acadêmica, pois, proporciona uma vivência importante quanto a elaboração de diagnóstico situacional, bem como, ensina sobre um olhar crítico e reflexivo da realidade vivenciada em uma UBS a partir de conhecimentos construídos em sala de aula. Para a UBS a construção do diagnóstico produz informações sobre estrutura física, agravos, dificuldades enfrentadas por usuários e profissionais o que poderá ser usado para planejar ações, afim, de melhorar os serviços ofertados (Perillo, 2020).

\section{Diagnóstico situacional da UBS}

A UBS do bairro da Ilha Grande de Santa Isabel comporta o módulo 01 que tem uma equipe formada por uma enfermeira, um médico, uma atendente de regulação, uma atendente social, uma técnica de enfermagem, um vigia, uma auxiliar de serviços gerais e nove Agentes Comunitários de Saúde (ACS). Essa UBS também comporta o módulo 02 que tem uma equipe formada por uma enfermeira, uma médica, uma atendente social, dois técnicos de enfermagem (um fica na sala de vacina e a outra realiza os procedimentos gerais), uma auxiliar de serviços gerais e nove ACS (Poças, Freitas \& Duarte, 2017).

A UBS tem uma recepção, um consultório odontológico (no momento, sem equipe de saúde bucal), dois corredores de espera, uma sala do médico, uma da enfermagem, uma para equipe multiprofissional (utilizada principalmente pela equipe de residência), uma farmácia que é unificada (a mesma farmácia para os dois módulos), uma sala de reunião, uma sala de procedimentos, uma sala de vacina, uma sala para armazenamento de prontuários, um almoxarifado e uma cozinha (Malta et al, 2017).

Fora da UBS e próximo a ela tem uma igreja católica (onde geralmente usam-se uma sala do lado para realização de um grupo de atividade coletiva), tem uma academia da praça, um prédio abandonado, mas que está em um ótimo estado de conservação (tratava-se de uma antiga superintendência, um anexo da prefeitura para atender algumas necessidades do bairro). Segundo a equipe da UBS, o bairro tem um Centro de Referência de Assistência Social (CRAS), quatro escolas de ensino fundamental, uma creche e algumas associações de moradores que trabalham com artesanato (Tasca, Massuda, Carvalho, Buchweitz \& Harzheim, 2020).

\section{Descrição da população atendida na UBS}

Com relação à população atendida pela UBS no sistema de informação são cadastrados 2.982 cidadãos. Definindo cada classe a seguir:

\section{- Gestantes (Gravidez na adolescência)}

São cadastradas 31 gestantes das quais em sua maioria são adolescentes. A gravidez na adolescência é uma realidade no território da Ilha. A quantidade de gestante com faixa etária entre 13 e 18 anos é bastante expressiva e isso chamou a atenção da equipe e requer uma atenção especial. Em uma conversa com algumas pessoas do próprio posto nos foi explicado que isso reflete um padrão de vida que as mesmas possuem, já advindo dos avós, onde ainda na juventude deixam de ir para escola para serem donas de casa. Devido a pandemia muitas gestantes demoram para iniciar o pré-natal o que leva ao o início tardio das consultas não garantindo uma atenção integral no acompanhamento da gestação o que pode dificultar muitas vezes a aceitação da gravidez, riscos de anemias entre outras complicações na gestação (Silva, De Paula, Ribeiro \& Brasil, 2021). 
- Saúde mental (grupo de escuta e acolhimento - GEA)

Existem 76 cidadãos com problemas de saúde mental. O Grupo de Escuta e Acolhimento - GEA surgiu a partir da necessidade de promoção de saúde, para além do modelo biomédico. Favorecendo a participação dos usuários, familiares e cuidadores no processo de promoção de saúde até mesmo na comunidade. A criação do grupo visava também uma administração correta e responsável dos psicotrópicos. O grupo funciona desde antes de 2010, com reuniões quinzenais, porém atualmente as reuniões se davam de forma semanal, o público do GEA, se dá desde crianças até idosos da comunidade Ilha Grande de Santa Isabel, contando com a participação dos profissionais, além da contribuição da Residência Multiprofissional e estagiários da saúde (Araújo et al, 2018).

Com o advento da pandemia as atividades coletivas foram suspensas, com isso os integrantes do GEA ficaram desassistidos, porém uma maneira encontrada de dar o mínimo suporte a esses usuários foi o atendimento espontâneo por meio daqueles que se dirigiam ao posto de saúde. Há também a ideia da criação de um grupo virtual para acompanhamento e liberação e orientação de atividades, a proposta do grupo ainda se encontra em andamento devido muitos usuários não possuírem acesso a meios virtuais (Coscrato \& Bueno, 2013).

\section{- Atividade Física e Práticas Corporais}

A UBS já contava com dois grupos de Atividade Física, "Mexa-se" e "Sob Medida", que estão inativos pela falta de profissionais capacitados que pudessem orientar e conduzir as práticas corporais. Contudo são grupos que serão reativados e terão como objetivo: reduzir a taxa de incidência de doenças e agravos não transmissíveis na população a partir da realização de exercícios semanais; estimular a população na realização de exercícios físicos orientados diariamente; diminuir intercorrências clínicas de doenças e agravos não transmissíveis a partir de exercícios físicos; diminuir gastos com saúde curativa, tendo-se um melhor custo-benefício com saúde preventiva; promover atividades educativas na comunidade, através de eventos da saúde; entre outros (Silva, Ono, Souza \& Menin, 2020).

\section{- Hipertensos e Diabéticos}

Com relação aos pacientes hipertensos eles correspondem um total de 336 e os diabéticos são 157 pacientes. Destacando as doenças crônicas não transmissíveis mais prevalentes e com grande taxa de causas de mortalidade no país. A hipertensão arterial (HA) é altamente prevalente nas populações ocidentais, facilmente identificável e passível de tratamento, consistindo em um dos fatores de risco mais importantes para a doença cardiovascular. E o diabetes mellitus (DM) é caracterizado por um distúrbio metabólico que consiste em hiperglicemia persistente, decorrente de deficiência na produção de insulina ou na sua ação, ou em ambos os mecanismos, ocasionando complicações em longo prazo (Marchetti \& Silva, 2020; Novello, 2017; Maeyama, 2020).

A prevalência da HA vem crescendo concomitantemente ao aumento da expectativa de vida e às mudanças no estilo de vida, com destaque para o crescimento do sobrepeso e da obesidade. E a DM atinge proporções epidêmicas, com estimativa de 415 milhões de portadores mundialmente. A persistência da hiperglicemia está associada a complicações crônicas micro e macrovasculares, aumentando a morbimortalidade e reduzindo a qualidade de vida (Novello, 2017; Maeyama, 2020).

\section{Conclusão}

O diagnóstico situacional é um dispositivo crucial de avaliação das Unidades Básica de Saúde sendo de total necessidade na formação de acadêmicos, pois, proporciona o entendimento dos problemas e as necessidades sociais como: necessidade de saúde, educação, saneamento, segurança, transporte, habitação, além de compreender a organização dos serviços de saúde. Através do diagnóstico situacional é possível planejar e direcionar as ações de saúde. sendo necessário 
conhecer a realidade, a dinâmica e os riscos que a população/comunidade está inserida e também a forma como estão organizados os serviços e as rotinas das unidades básicas de saúde e das equipes da UBS. Portanto, esse dispositivo é de fundamental importância para o levantamento de problemas da UBS, que por sua vez fundamenta o planejamento estratégico situacional que permite desenvolver ações de saúde mais focais efetivas em relação aos problemas encontrados. Nesse sentido, o presente estudo pode ajudar no desenvolvimento das políticas públicas de saúde com foco na prevenção, orientação e tomada de medidas que melhorem os indicadores de saúde.

\section{Referências}

Araujo, G. R., Monteiro, F. S. C. T., Machado, C. S. (2018). Grupo de escuta e acolhimento-GEA: uma ferramenta de cuidado em saúde mental na estratégia de saúde da família-esf. Infinitum: revista multidisciplinar 1(1):69-89.

Brasil. Ministério da Saúde. Secretaria de Atenção à Saúde. (2017). Portaria no 2.436, de 21 de setembro de 2017. Aprova a Política Nacional de Atenção Básica, estabelecendo a revisão de diretrizes para a organização da Atenção Básica, no âmbito do Sistema Único de Saúde (SUS). Diário Oficial da União. Brasília, DF.

Coscrato, G. \& Bueno, S. M. V. (2013). Concepts of health education by public health nurses. Revista da Escola de Enfermagem da USP, 47(3):714-721. Gil, A. C. (2007) Como Elaborar Projetos de Pesquis., Atlas, 4(4): 41-57.

Maeyama, M. A. (2020). Aspectos relacionados à dificuldade do controle glicêmico em pacientes com Diabetes Mellitus tipo 2 na Atenção Básica. Brazilian Journal of Development, 6(7): 47352-47369.

Malta, D. C., Bernal, R. T. I., Lima, M. G., Araújo, S. S. C., Silva, M. M. A., Freitas, M. I. de F. \& Barros, M. B. A. (2017). Noncommunicable diseases and the use of health services: Analysis of the National Health Survey in Brazil. Revista de Saúde Publica, 51, 1S-10S

Marchetti, J. R. \& da Silva, M. (2020). Educação em saúde na atenção primária: Diabetes Mellitus. Anuário Pesquisa E Extensão Unoesc Xanxerê, 5, e24183.

Novello, M. F. (2017). Conformidade da Prescrição Anti-Hipertensiva e Controle da Pressão Arterial na Atenção Básica. Arquivos Brasileiros de Cardiologia, 108(2):135-142.

Paim, J. S. Sistema Único de Saúde (SUS) aos 30 anos (2018) Ciênc. saúde coletiva, 23(6):1723-1728.

Perillo, R. D., Poças, K. C., Machado, I. E., Bernal, R. T. I., Duarte, E. C. \& Malta, D. C. (2020). Fatores associados à utilização da atenção primária pela população adulta de Belo Horizonte, Minas Gerais, segundo inquérito telefônico. Revista Mineira de Enfermagem, 24:1-10.

Poças, K. C., Freitas, L. R. S. de, \& Duarte, E. C. (2017). Censo de estrutura da Atenção Primária à Saúde no Brasil (2012): estimativas de coberturas potenciais. Epidemiologia e serviços de saúde, 26(2): 275-284.

Sacramento, I. (2018). A saúde numa sociedade de verdades. Revista Eletrônica de Comunicação, Informação e Inovação em Saúde, v.12, n.1.

Silva, E. S. M., Ono, B. H. V. S., Souza, J. C. \& Menin, I. B. F. (2020). Media and health promotion in times of COVID-19. Research, Society and Development, 9(8):842986252.

Silva, C. V., Pinheiro, F. A., Batista T. L., Guerra, N. A. Q., Souza, T. V. T. S \& Gomes R. K. G. (2019). Educação em saúde na atenção primária: aplicação de um panfleto informativo sobre febre amarela. Mostra Interdisciplinar Do Curso De Enfermagem, 3(1).

Silva, E. A. \& Terencio, M. L. (2019). Educação em Saúde: a utilização de panfletos informativos e educativos como estratégia de educação e prevenção às patologias mais prevalentes da população no contexto da atenção básica [Artigo completo]. In Universidade Federal da Integração Latino-Americana Artigos Científicos, II Semana Integrada de Ensino, Pesquisa e Extensão 217-221.

Sistema Único de Saúde (SUS): Estrutura, princípios e como funciona. http://www.saude.gov.br/sistema-unico-de-saude.

Tasca, R., Massuda, A., Carvalho, W. M., Buchweitz, C., \& Harzheim, E. (2020). Recommendations to strengthen primary health care in Brazil. Revista Panamericana de Salud Publica, 44.

Silva, S. S. F, De Paula, D. G., Ribeiro, M. M. \& Brasil, Y. Z. (2021). Comunicação em saúde por uma população adscrita de uma clínica da família do município do Rio de Janeiro. Research, Society and Development, 10(4):45810414279. 